EPJ Web of Conferences 59, 13011 (2013)

DOI: $10.1051 /$ epjconf/20135913011

(C) Owned by the authors, published by EDP Sciences, 2013

\title{
Multichannel down-scattered neutron detector for areal density measurement
}

\author{
Y. Arikawa ${ }^{1}$, H. Hosoda ${ }^{1}$, T. Nagai ${ }^{1}$, K. Watanabe ${ }^{1}$, K. Yamanoi ${ }^{1}$, \\ M. Cadatal-Raduban ${ }^{1}$, T. Shimizu ${ }^{1}$, N. Sarukura ${ }^{1}$, M. Nakai ${ }^{1}$, T. Norimatsu ${ }^{1}$, \\ H. Azechi ${ }^{1}$, N. Izumi ${ }^{2}$, T. Murata ${ }^{1,3}$, T. Suyama ${ }^{4}$, A. Yoshikawa ${ }^{5}$, K. Kamada ${ }^{6}$, \\ Y. Usuki ${ }^{6}$, N. Satoh ${ }^{7}$ and H. Kan ${ }^{7}$ \\ 1 Institute of Laser Engineering, Osaka University, 2-6 Yamadaoka, Suita, Osaka 565-0871, \\ Japan \\ 2 Lawerence Livermore National Laboratory, Livermore, California 94550, USA \\ 3 Kumamoto University, 2-40-1 Kurokami, Kumamoto 860-8555, Japan \\ 4 Tokuyama Corporation, 3-2-1 Kasumigaseki, Chiyoda-ku, Tokyo 100-8983, Japan \\ 5 Tohoku University, 2-1-1 Katahira, Aoyou, Sendai, Miyagi 980-8577, Japan \\ ${ }^{6}$ Furukawa Co., Ltd., 1-25-13, Kannondai, Tsukuba, Ibaraki 305-0856, Japan \\ 7 Hamamatsu Photonics k.k., 1820, Kurematsu, Nishi-ku, Hamamatsu-City 431-1202, Japan
}

\begin{abstract}
A down-scattered neutron detector operating in the multichannel counting mode was developed for areal density $(\rho \mathrm{R})$ measurement. Equipped with a newly developed ${ }^{6} \mathrm{Li}$ glass scintillator (APLF80), the detector was tested in a fusion experiment at the GEKKO XII facility, Osaka University, Osaka, Japan. For a low- $\rho$ R fusion shot, the detector clearly discriminated the $\gamma$-rays, primary neutrons, $\gamma$-rays produced via $(\mathrm{n}, \gamma)$ reactions from the target chamber, and neutrons scattered by the target chamber. Furthermore, the observed signal was in good agreement with predictions made by Monte Carlo simulation.
\end{abstract}

\section{INTRODUCTION}

Down-scattered neutron diagnostics is one of the most promising methods for measuring the areal density $(\rho \mathbf{R})$ of the imploded core in an inertial confinement fusion experiment. With this method, the $\rho \mathbf{R}$ can be calculated from the ratio of unscattered (primary) to scattered neutrons within dynamic ranges of up to $3 \mathrm{~g} / \mathrm{cm}^{2}$ [1]. The first successful demonstration of $\rho \mathrm{R}$ measurement using down-scattered neutrons was reported at the IFSA 2011 conference by the neutron diagnostics team from the National Ignition Facility (Lawrence Livermore National Laboratory, Livermore, CA, USA), who used the Magnetic Recoil Spectrometer [2] and a neutron time-of-flight detector with a plastic scintillator [3]. However, the main limitation of such a detection method is that the low-intensity down-scattered neutrons only arrive at the detector after a very high intensity primary neutron "flash." Thus, the detector must have a faster response and a more selective, higher sensitivity for down-scattered neutrons, particularly in the lower$\rho$ R and lower-neutron-yield experiments at GEKKO XII (Osaka University, Osaka, Japan) and OMEGA (Laboratory for Laser Energetics, Rochester, NY, USA). Furthermore, such an improved detector is a key requirement for the detection of down-scattered neutrons from deuterium-deuterium (DD) fusion in the ongoing fast ignition realization experiments (FIREX) at the Institute of Laser Engineering, Osaka University.

This is an Open Access article distributed under the terms of the Creative Commons Attribution License 2.0, which permits unrestricted use, distribution, and reproduction in any medium, provided the original work is properly cited. 


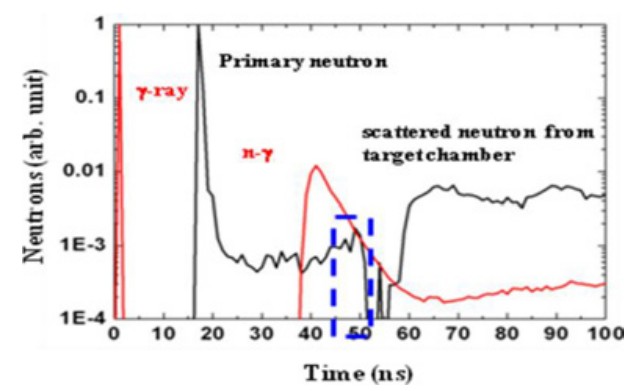

Figure 1. Simulated $\gamma$-rays and neutron signals taking into account ${ }^{6} \mathrm{Li}$ scintillator detection efficiency using the MCNP5 Monte Carlo simulation code for $\rho \mathrm{R}=0.1 \mathrm{~g} / \mathrm{cm}^{2}$ and a carbon-deuterium (CD) core.

For these purposes, a fast-response ${ }^{6} \mathrm{Li}$ glass scintillator (APLF80) has been developed [4]. The energy peak of the down-scattered neutrons from DD fusion is at $0.27 \mathrm{MeV}$, matching the resonant peak in the cross section of neutron detection for a ${ }^{6} \mathrm{Li}$ scintillator [1]. Moreover, a ${ }^{6} \mathrm{Li}$ scintillator can produce sufficient photons for detection from low-energy neutrons via an exothermic reaction $\left({ }^{6} \mathrm{Li}(n, t) \mathrm{He}+\right.$ 4.8 MeV), thus realizing selective, high sensitivity for down-scattered neutrons [1]. However, it is still very difficult to sufficiently suppress the primary neutron flash.

A powerful solution to the flash problem is the use of the multichannel counting mode [1]. Multichannel counting with a sufficiently large number of very small scintillator pixels will reduce the primary neutron flash per pixel while also ensuring that the down-scattered neutrons are detected by at least some pixels. Thus, a down-scattered neutron detector operating in multichannel counting mode using an APLF80+3Pr scintillator array was developed for GEKKO XII. This paper presents the details of the detector and the results of performance tests performed at GEKKO XII.

\section{DETECTOR DESIGN AND EXPERIMENTAL SETUP}

APLF80+3Pr glass was cut and polished into quadrangle prisms with the dimensions $2 \mathrm{~mm} \times 2 \mathrm{~mm}$ $\times 20 \mathrm{~mm}$ and assembled into a scintillator array with 400 pixels using $\mathrm{BaSO}_{4}$ reflection paint [5]. This scintillator array was attached to a multianode photomultiplier tube (H9500 Hamamatsu, 256 channels with the pixel size of $3 \mathrm{~mm}$ ) for this study (and will be upgraded to 400 channel in the future). The 256 anode channels simultaneously were recorded with time-to-digital convertors (TDCs). Two anode signals were monitored by an oscilloscope. Crosstalk between channels was checked experimentally using a sealed neutron source; the signal from a single neutron was confirmed to be detected by four neighboring channels. However, when the array of signals is used to reconstruct an image, a neutron is detected as an independent spot.

The detector was installed at GEKKO XII with the scintillator located $36 \mathrm{~cm}$ from the target. In this geometry, the $\gamma$-rays, primary neutrons (P-N), down-scattered neutrons (DS-N), and scattered neutrons from the target chamber are detected in the time series, as shown in Fig. 1.

A 4-cm-thick lead shield was placed in front and a 1-cm-thick shield around the detector to block $\gamma$-rays such as those originating from the $(n, \gamma)$ reaction at the target chamber wall (hereinafter referred to as $n-\gamma)$. The pulse height from a DD neutron was confirmed to be about $60 \mathrm{mV}$, and the threshold of the TDC was set to $50 \mathrm{mV}$. The detection sensitivity was experimentally calibrated in the GEKKO XII fusion experiment, and it showed a good agreement with the design. An exploding pusher shot with a $3-\mu$ m-thick $C D$ shell produced $3 \times 10^{8}$ neutrons, and the $\rho \mathrm{R}$ was measured from secondary reaction diagnostics to be $6-11 \mathrm{mg} / \mathrm{cm}^{2}$ by MANDALA, a multichannel neutron scintillator array [6]. As shown in Fig. 2(a), the primary neutron signals are seen at $20 \mathrm{~ns}$ with a pulse height of $300 \mathrm{mV}$, which corresponds to about five neutron counts. The down-scattered neutron edge arrival time is $50 \mathrm{~ns}$. 
(a)

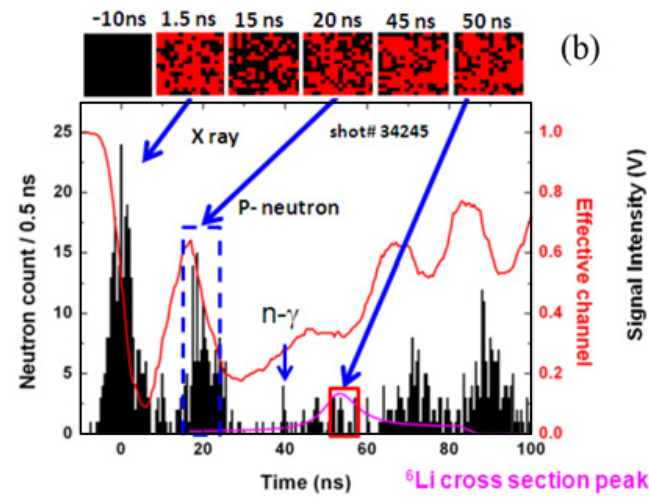

(b)

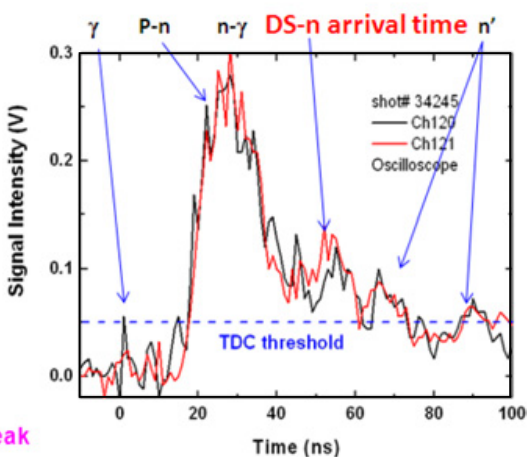

Figure 2. (a) Observed neutron count history (left axis) and effective channel (right axis) at the down-scattered neutron detector for the exploding pusher shot, and (b) the picked up waveform. The scintillation images were sampled every $500 \mathrm{ps}$.



Figure 3. Comparison of experimental and simulated neutron counts for various $\rho$ R. The right side shows a zoomed-up view along the vertical axis. The experimental and calculated neutrons were summed every $3 \mathrm{~ns}$, which is the detection time spread for $0.27-\mathrm{MeV}$ neutrons in the scintillator.

The neutron signal in Fig. 2(b) shows the possibility that this is a down-scattered neutron. The experimental neutron counts were corrected on the basis of the portion of effective channels operating each time. In the calculation models, we assumed solid CD at room temperature with the various $\rho \mathrm{R}$ values, a detector with a lead shield to scale, and a target chamber. Further, the experimental signal counts of the primary neutrons detected within 15-21 ns were normalized with the simulated values. As shown in Fig. 3, the experimentally observed signal fits very well with the simulation at around 30 and $60 \mathrm{~ns}$. However, the down-scattered neutron signal at $50 \mathrm{~ns}$ fits the line of $\rho \mathrm{R}=800 \mathrm{mg} / \mathrm{cm}^{2}$, which is unrealistically high. This signal is considered to be partially contained signal from $n-\gamma$. Actually, the $n-\gamma$ peak is seen clearly at $35 \mathrm{~ns}$, as expected. This can be improved by upgrading a shield.

The present limitations of the developed detector were clearly elucidated by this experiment: they are $n-\gamma$ and the TDC dead time. To address these, the detector will be modified as follows: (1) it will be placed at $15 \mathrm{~cm}$ from the target in order to ensure $n-\gamma$ arrival after the down-scattered neutron edge, and (2) the TDCs will be replaced with faster analogue-to-digital convertors (ADC) to record the waveforms on all channels.

Measurement of the $\rho \mathrm{R}$ in a fast ignition shot is one of most desired objectives in our FIREX project. In 2010, a neutron yield (Yn) of $5 \times 10^{6}$ was observed from a fast ignition target imploded by 12 beams 
(a)

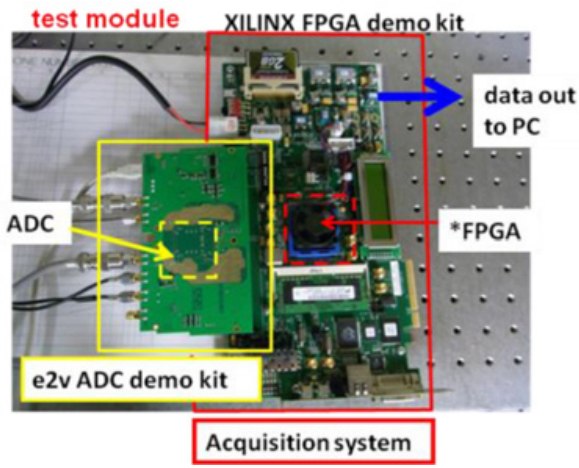



Figure 4. (a) Photograph of testing module for fast ADC. (b) Comparisons of detected signals from a pulse generator.

without a heating laser. (Note that the fast ignition experiment with heating generates very intense $\gamma$-rays and neutrons via $\mathrm{D}(\gamma, \mathrm{n}) \mathrm{p}$ from the target, and so detection without heating is our target for the developed detector.) In this shot, the modified design target was tested with the aim of obtaining a $\rho \mathrm{R}$ greater than the previously reported [7] value of $200 \mathrm{mg} / \mathrm{cm}^{2}$, although this value was not measured in that experiment. With our improved detector, three counts of down-scattered neutrons at the back scattering edge can be expected from the $\mathrm{Yn}$ of $5 \times 10^{6}$ and $\rho \mathrm{R}$ of $200 \mathrm{mg} / \mathrm{cm}^{2}$.

\section{FUTURE HARDWARE DEVELOPMENTS}

A fast multichannel ADC system is being developed for this purpose, as shown in Fig. 4(a), by e2v in France. The ADC demonstration kit developed by e2v (on the left in Fig. 4(a)) includes a fast quad-ADC with a bandwidth $(-3 \mathrm{~dB})$ of $2 \mathrm{GHz}$ and sampling rate of $1.25-5 \mathrm{GSPS}$ per channel. The acquisition system (on the right side in Fig. 4(a)) consists of a multipurpose development kit with a field-programmable gate array controlled by a PC via USB cables. Comparisons of the detected signals with 1-GHz digital oscilloscopes (Fig. 4(b)) showed the excellent performance of the system. An ADC powered by an NIM standard unit with an 8-channel analogue input and an external trigger input will be developed with an estimated cost per unit of $\$ 6500$. The 256 channels of the ADC will be installed in the next fast ignition experiment planed for 2012.

\section{SUMMARY}

A down-scattered neutron detector equipped with a new developed ${ }^{6} \mathrm{Li}$ glass scintillator (APLF80) that can operate in multichannel counting mode was developed for areal density measurement. The detector was demonstrated in a fusion experiment at the GEKKO XII facility, Osaka University. An exploding pusher shot with a $\rho \mathrm{R}$ of $6-11 \mathrm{mg} / \mathrm{cm}^{2}$ was tested, but signals corresponding to $\rho \mathrm{R}=800 \mathrm{mg} / \mathrm{cm}^{2}$ was detected. The $\gamma$-rays, primary neutrons, $\gamma$-rays from $(\mathrm{n}, \gamma)$, and neutrons scattered by the target chamber were discriminated. The observed signal showed good agreement with the predictions of a Monte Carlo simulation. The dead time of the TDC is one of the critical issues in the current system. A multi channel ADC is being developed to detect the down-scattered neutrons in the fast ignition experiment. 


\section{IFSA 2011}

\section{References}

[1] N. Izumi, et al., Rev. Sci. Instrum. 74, 3, 1722 (2003)

[2] J.A. Frenje, et al., Phy. Plasmas 17, 056311 (2010)

[3] V. Yu. Glebov, et al., Rev. Sci. Instrum. 81, 10D325 (2010)

[4] Y. Arikawa, et al., Rev. Sci. Instrum. 80, 113504 (2009)

[5] Y. Arikawa, et al., Rev. Sci. Instrum. 81, 10D303 (2010)

[6] N. Izumi, et al., Rev. Sci. Instrum. 70, 1, 1221 (1999)

[7] R. Kodama, et al., Nature 412, 23 (2001) 